\title{
Evidence of magma activation beneath the Harrat Lunayyir basaltic field (Saudi Arabia) from attenuation tomography
}

\author{
I. Koulakov ${ }^{1,2}$, S. El Khrepy ${ }^{3,4}$, N. Al-Arifi ${ }^{3}$, I. Sychev ${ }^{1}$, and P. Kuznetsov ${ }^{1}$ \\ ${ }^{1}$ Trofimuk Institute of Petroleum Geology and Geophysics SB RAS, Prospekt Koptyuga, 3, 630090, \\ Novosibirsk, Russia \\ ${ }^{2}$ Novosibirsk State University, Pirogova 2, 630090, Novosibirsk, Russia \\ ${ }^{3}$ King Saud University, P. O. Box 2455, Riyadh 11451, Saudi Arabia \\ ${ }^{4}$ National Research Institute of Astronomy and Geophysics, NRIAG, 11421, Helwan, Egypt \\ Correspondence to: I. Koulakov (ivan.science@gmail.com)
}

Received: 7 May 2014 - Published in Solid Earth Discuss.: 11 June 2014

Revised: 25 July 2014 - Accepted: 25 July 2014 - Published: 27 August 2014

\begin{abstract}
We present a seismic attenuation model for the crust beneath the Cenozoic basaltic field of Harrat Lunayyir (western Saudi Arabia), where a strong seismic swarm occurred in 2009. The tomography inversion uses the envelope shape of the $\mathrm{S}$ wave seismograms from over 300 strong events $(M<3.5)$. The resulting attenuation structures appear to be consistent with the distribution of seismic velocities. The obtained 3-D attenuation model distinguishes the low-attenuation zones down to $5 \mathrm{~km}$ depth corresponding to the rigid basaltic cover. At greater depths, we detect a high-attenuation anomaly coinciding with the main seismicity cluster. We propose that this zone corresponds to the upper part of the conduit area ascending from deeper magma sources. According to the distributions of local events, fluids and melts from this conduit appear to reach a depth of $\sim 2 \mathrm{~km}$, but were not able to reach the surface and cause the eruption in 2009.
\end{abstract}

\section{Introduction}

The western part of the Arabian Peninsula is characterized by a wide distribution of Cenozoic basaltic fields (harrats). Some of these fields are still active and present a real eruption potential. Throughout history there have been several records of eruptions within the harrats of Saudi Arabia and Yemen (e.g., Camp et al., 1987). The harrats are located close to the western coast of the Arabian Peninsula, and their appearance is thought to be linked to the rifting of the Red
Sea (e.g., Cochran and Martinez, 1988). However, the role that the opening of the Red Sea played in the origination of Harrat Lunayyir and other harrats appears to be rather complex. Unlike the southern Red Sea segment, which has a clear ocean-type spreading, the extension of the northern segment appears to result from the stretching of the continental crust at a rate of $\sim 10 \mathrm{~mm} \mathrm{year}^{-1}$ (Cochran and Karner, 2007). According to regional tomography studies (e.g., Chang et al., 2011), no low-velocity anomalies, which could be associated with hot areas, have been observed beneath the Red Sea. Instead, negative seismic anomalies align beneath the western part of the Arabian Plate exactly where the volcanic fields are located. These observations may give arguments for the passive character of rifting in the northern Red Sea. However, the lithosphere extension may cause the asthenosphere upwelling beneath the coastal areas that is responsible for the volcanic activity.

Lunayyir is one of the youngest harrats in Saudi Arabia (less than $1 \mathrm{Ma}$, Pallister et al., 2010), and it is small relative to other Cenozoic basaltic fields in the Arabian Peninsula. In Lunayyir, recent basalts cover an area that is over $3500 \mathrm{~km}^{2}$ in size (Fig. 1b). The last eruption in this area occurred approximately 1000 years ago (Camp et al., 1987); however, elevated groundwater temperatures and the existence of fumaroles indicate that strong thermal activity still occurs in this zone (e.g., Al-Dayel, 1988). This volcanic field is considered a probable candidate for the next eruption. Before 2009, weak-to-moderate seismicity mostly associated with tectonic crustal displacements in the Red Sea and Gulf of 

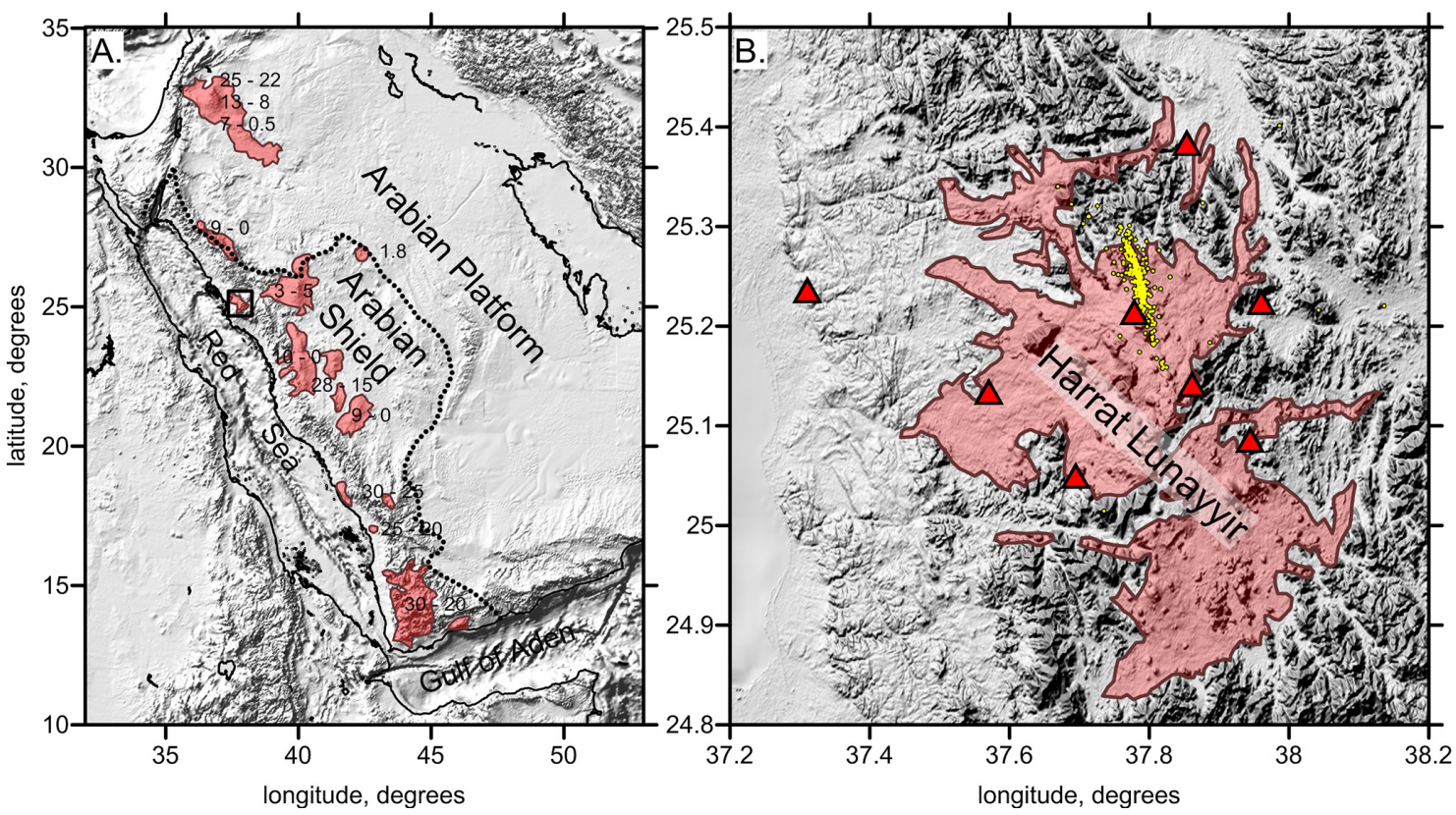

Figure 1. Study region in the framework of regional tectonics and data distribution. Topographic map of the Arabian Peninsula (a). The dotted line indicates the boundary between the Arabian Shield and the Arabian Platform. The reddish spots mark the Cenozoic basaltic fields (harrats) with their activity ages. The black rectangle is the location of the Harrat Lunayyir. Detail of the study area (b). The triangles show the seismic stations and the yellow dots mark the event locations used in this study.

Aqaba was observed in this area (e.g., El-Isa and Al-Shanti, 1989; Al-Amri, 1995). In April-July 2009, a strong swarm of over 30000 earthquakes reaching a magnitude of $5.4 \mathrm{oc}-$ curred in the Lunayyir area. Most of these events were confined to have been oriented along a NNW-SSE line that was approximately $20 \mathrm{~km}$ long in the northern part of the harrat (Fig. 1b). This seismicity included both high- and lowfrequency events (Pallister et al., 2010) that might indicate the potentially volcanic nature of this swarm. This hypothesis was supported by models based on interferometric synthetic aperture radar (InSAR) and geodetic data developed by Baer and Hamiel (2010), who proposed a vertical dyke intrusion.

Questions relating to the origin of this swarm and the possible continuation of these processes lead to vital discussions in the scientific community. Studying the deep structure beneath the Lunayyir area can greatly help with understanding these problems. The spatial and temporal distributions of the seismicity and earthquake mechanisms were carefully studied by Zobin et al. (2013). They compared the main seismic activity features to several volcanoes around the world and concluded that further volcanic activity was not plausible in the Lunayyir area. However, most other authors studying different aspects of this problem have suggested future volcanic intrusions are likely. In particular, the recent seismic tomography study by Hansen et al. (2013) discovered a high- $P$ velocity body that was interpreted as an area with repeated magmatic intrusions. This result was obtained based on the accurate, double-difference location of over 5000 seismic events and the tomographic inversion of $P$ wave arrival times from local earthquakes. At the same time, the local earthquake tomography suffered a trade-off between the velocity structure and the source parameters, which should be simultaneously determined during the inversion. With highly concentrated source locations, such as those found beneath the Lunayyir area, the velocity around the seismicity cluster might appear to be poorly resolved because of trade-offs among the seismic velocity, origin time, and event depth. When only $P$ data are used, the situation worsens. Therefore, we propose that the results of Hansen et al. (2013) should be carefully tested in independent studies using different methods.

In this study, we present attenuation tomography results for the same area studied by Hansen et al. (2013). The advantage of attenuation tomography over the local earthquake velocity tomography is that it addresses the linearized inverse problem, which affects neither the source localization nor the ray paths and requires only one inversion iteration. For slightly mislocated sources, the location of the retrieved attenuation patterns can be slightly biased; however, this effect generally appears minor during interpretation. In addition, the attenuation parameters are more sensitive to the fracture distribution and liquid phase content (fluids and melts), which seem to be important for determining the origin of the Lunayyir activation.

Seismic tomography is a powerful tool for studying magmatic sources beneath volcanic areas (see, for example, 
overviews in Lees, 2007, and Koulakov, 2013, which present several successful examples of tomography studies of volcanoes). Attenuation tomography has been successfully used in many studies of crustal and mantle structures. Regional attenuation structures in the mantle beneath the Arabian Plate surrounding the studied region were investigated together with Pn and Sn velocities by Al-Damegh et al. (2004). They found a presence of a large mantle anomaly beneath western side of the Arabian Plate which is interpreted as a zone of thinner lithosphere and hot asthenosphere. The attenuation tomography was used for studying crustal structures beneath volcanoes in different regions using active source data, such as in the case of Medicine Lake in California (Evans and Zucca, 1988), and passive source data, including Loma Prieta (Lees and Lindley, 1994), Etna (Martínez-Arévalo et al., 2005), central Andes (Shurr et al., 2003, Koulakov et al., 2006), and Kilauea (Hansen et al., 2004). In the case of dormant volcanoes, these studies generally identified lower-attenuation zones beneath volcanoes, whereas in the cases of active volcanoes, they found contrasted patterns of higher attenuation. Most of these studies used a technique based on retrieving $t^{*}$ by analyzing waveforms around $P$ wave arrivals. The $t^{*}$ values of the $S$ waves at different frequencies were used in some studies, such as the tomographic inversion of the North Anatolian fault (Koulakov et al., 2010). A similar approach was used in the present study; however, instead of using $t^{*}$, we estimated the attenuation parameter by analyzing the envelope shape of the $S$ wave signal, as proposed in the classic work by Aki and Chouet (1975). In this study, we provide the seismic attenuation distribution beneath the Lunayyir area, which helps us understand the origin of recent seismic activities.

\section{Data and algorithms}

After the beginning of the seismic crisis in April 2009, a seismic network was installed in the Lunayyir area by the King Abdulaziz City for Science and Technology (KACST), King Saud University (KSU), and the Saudi Geological Survey (SGS). Data for over 5700 events using auto-picked $P$ arrival times were used by Hansen et al. (2013). In this study, we manually re-analyzed the same waveform data. We selected 1879 strong events with the magnitudes above 3.2 and identified $8904 P$ and $10579 S$ phases for most of these events at the available stations, which enabled the robust locating of the sources. It is interesting that there were slightly more $S$ phases than $P$ phases, which can be explained by the clearer and stronger $\mathrm{S}$ wave signal. These data were used to run a simultaneous tomographic inversion for $P$ and $S$ velocities and source locations based on the iterative LOTOS (Local Tomography Software) algorithms (Koulakov, 2009). The results of the velocity inversion are described in detail in Koulakov et al. (2014).
The resulting velocity model, which is presented in Fig. 2, is used in this study as the background model for the attenuation tomography.

After performing the source locations, we analyzed the seismograms of every source-receiver pair to estimate the attenuation parameters of each ray. We used the basic attenuation estimation principles proposed in the classic work of Aki and Chouet (1975). The physical background of the method is schematically illustrated in Fig. 3. We are studying the intrinsic attenuation, which is responsible for conversion of a part of the seismic energy into the heat and leads to the decrease of the amplitude of seismic signal on the seismogram. However, directly deriving the attenuation values from the measurement of the amplitude of seismic signal is an ambitious task, because the amplitude may be also affected by a variety of different factors, such as source characteristics, geometrical spreading, focusing/de-focusing, scattering, and reflections. Studying the properties of seismic signal following the main wave arrival (seismic coda) provides more information on the medium intrinsic attenuation. For example, striking a piece of metal with low attenuation yields a sound that is affected by the existence of numerous scatterers and reflectors that can be heard for a rather long time (long flat coda). Alternatively, sound from a highly attenuated wooden balk would be much shorter (short coda). When studying earth structures, it is presumed that the medium contains a large amount of scatterers which are more or less uniformly distributed throughout the space (hatches in Fig. 3). In this case, besides the direct wave there are an infinite number of secondary scattering waves which compose the coda. The properties of the coda depend on the properties of the medium in which the main and scattered waves propagate. In the case of a highly attenuated medium (e.g., corresponding to station B in Fig. 3), more energy of the seismic waves will be converted into the heat and this causes stronger decay of the envelop amplitude compared to the direct wave. For the low-attenuation medium (case of station A), the coda decrement is longer. Aki and Chouet (1975) approximated the envelope for seismic coda after the arrival of the main wave using an exponential dependence:

$A(f, t)=t^{-\beta} A_{0} \exp \left(\frac{-\pi f t}{Q(f)}\right)$,

where $t$ is travel time, $A_{0}$ is the signal amplitude in the source area, $f$ is its frequency, and $Q(f)$ is a quality factor (inverse of the attenuation). Figure 4 a shows a real seismogram corresponding to the horizontal component filtered at $3 \mathrm{~Hz}$. The envelope of the waveform is shown in Fig. 4b. Applying a logarithmic transformation and then smoothing (central moving average in a $2 \mathrm{~s}$ interval) provides the curve shown in Fig. 4c, which can be approximated using a straight line directly associated with the attenuation:

$\ln (A(f, t))+\beta \ln (t)=\ln \left(A_{0}\right)-\frac{-\pi f t}{Q(f)}$. 

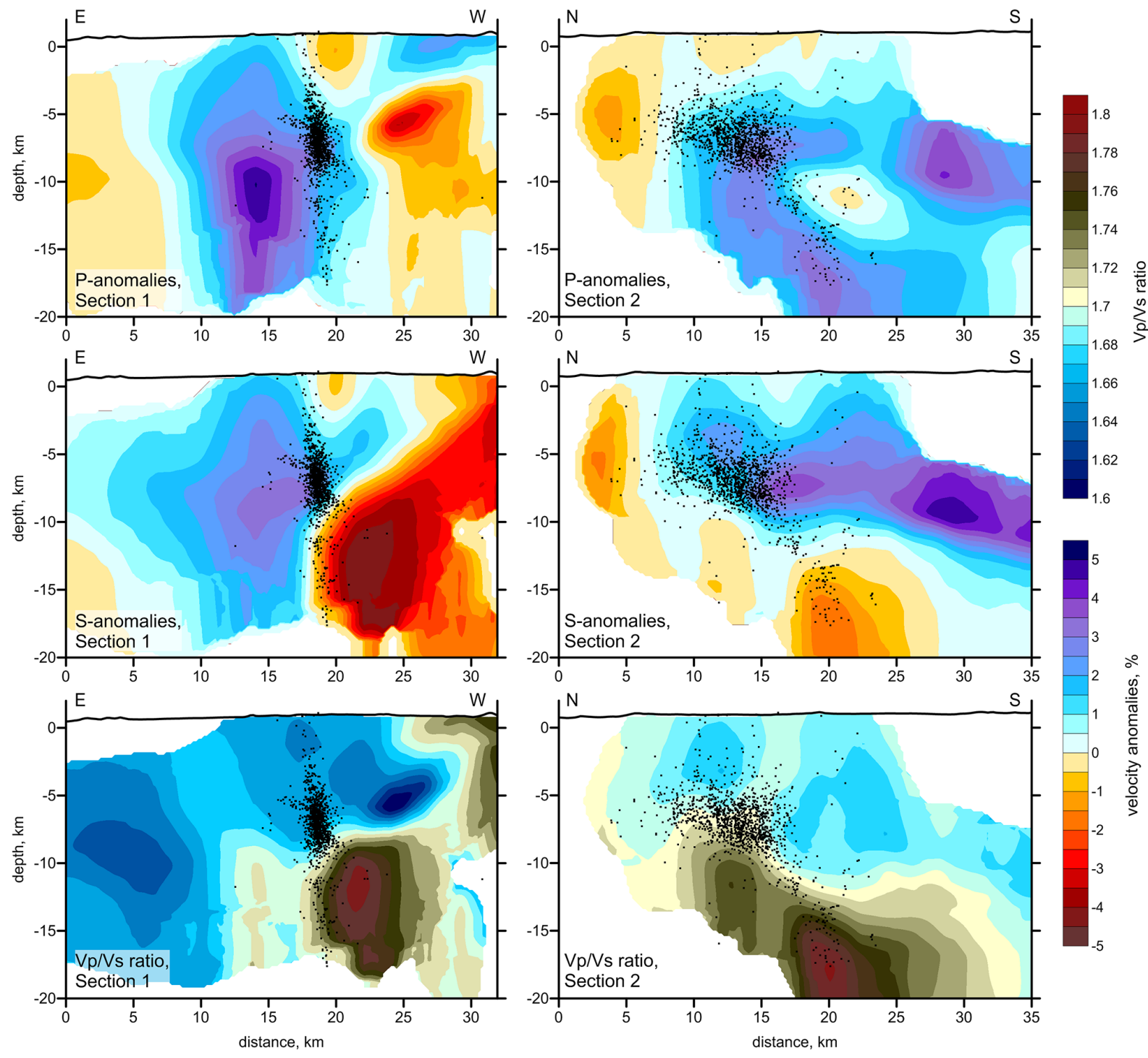

Figure 2. Velocity model ( $P, S$ anomalies and Vp / Vs ratio) and local source hypocenters (black dots) from Koulakov et al. (2014) in two vertical sections with the same locations as shown in Fig. 7. These model and source coordinates were used to construct the rays for the attenuation tomography in this study.

The effect of geometrical spreading can be easily estimated using existing seismic ray parameters in the existing velocity model. Thus, the slope of the fit line shown in Fig. $4 \mathrm{c}$ is proportional to the attenuation value, $1 / Q$. For example, a gentle slope (long coda) indicates a high-quality factor and low attenuation. To avoid small numbers in the output from the tomography inversion, we use the inverse of the attenuation multiplied by 1000 . Because of the uncertainty in $A_{0}$, we cannot obtain an absolute value for the quality factor. Therefore, we use the average estimated values as a reference for the quality factor. We computed the relative positive and negative deviations in the quality factor for the inversion with respect to the reference value.
In the analysis of seismograms, we consider a $10 \mathrm{~s}$ lapse window starting at $1 \mathrm{~s}$ after the arrival of the $S$ phase. This is different than the common way for computing the slope of the Q-coda which is usually based on the two travel times of the $\mathrm{S}$ wave as a starting point (e.g., Aki, 1980; Rautian and Khalturin, 1979). We could not use the traditional definition of coda because, for most events, the signal was not very strong. After the moment of two $S$ travel times, the low signal-to-noise ratio made the determinations of the coda characteristics very unstable. The delay of $1 \mathrm{~s}$ seems to us optimal to start the lapse window as corresponding to the maximum amplitude of the direct waves. Just after the first arrival, the shape of the seismogram is dependent on the source properties and usually does not reach the maximum immediately. 


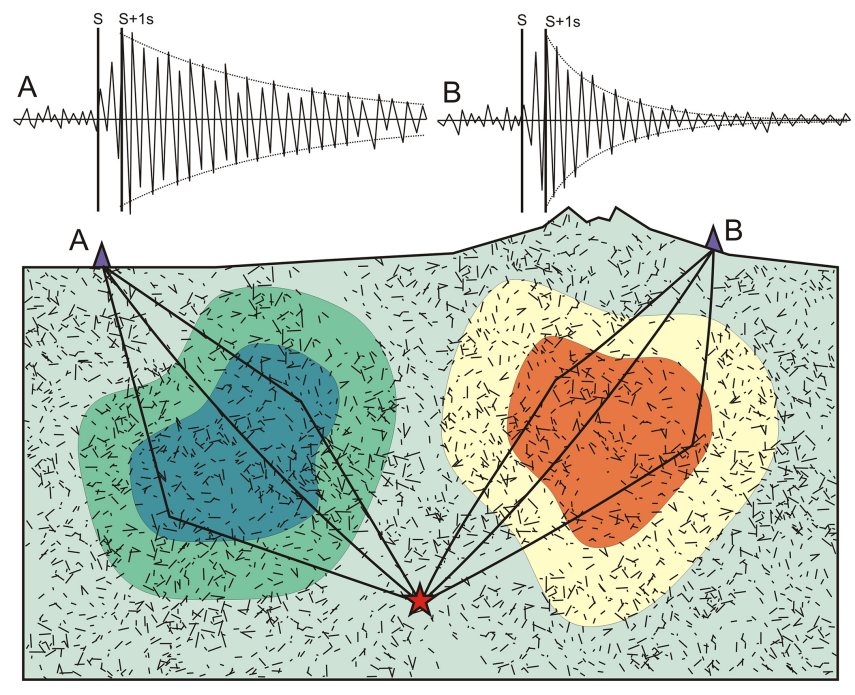

Figure 3. Schematic representation of the basic principle used for the attenuation inversion in this study. Hatches are the scatterers which are roughly uniformly distributed within the study area. Red and blue indicate zones of high and low attenuation, respectively. The star is a source; triangles are the receivers; black lines are the direct and scattered rays between source and receivers. Above: schematic seismograms recorded at stations A and B corresponding to low- and high-attenuation areas. Dotted lines are the envelopes of the seismograms. Lines indicated with $S$ and $S+1$ s mark the arrival time of the $\mathrm{S}$ wave and $1 \mathrm{~s}$ later point.

In our experience, the best exponential approximation for the coda decrement can be obtained for the window starting close to the moment of maximum of the direct wave. Comparing the amplitudes of direct and secondary waves seems to be more stable than the analysis of only secondary waves, which are strongly affected by many different factors (such as distribution of scatterers). We made several attempts at using other criteria for defining the start of the lapse times (such as two $S$ times) but obtained unstable solutions which were inconsistent for different frequencies. In this sense, here we do not implement a commonly used estimate for slope of the Q-coda, but the measurement of how large the S wave packet is compared to the coda waves (for a wide discussion on this topic, see Sato and Fehler, 1998 and Gusev and Abubakirov, 1999). In other words, this Q-coda parameter matches more to the measure of the $\mathrm{S}$ wave envelope broadening than directly to the estimation of the attenuation parameter along the ray path.

The data were independently processed across narrowband-filtered seismograms with six different frequencies: $0.75,1.50,3,6,12$, and $24 \mathrm{~Hz}$. The processing results were carefully visually inspected for each ray. As a result, many data were rejected for not providing a clear linear approximation; only 4326 values had a sufficiently high quality for the inversion.
The attenuation tomographic inversion algorithm was generally identical to the first iteration step of the LOTOS code (Koulakov, 2009), which was initially designed for travel time passive source tomography. In our case, the source locations were obtained at a previous step of velocity tomography and remained unchanged when computing the attenuation. The rays were constructed using a bending ray tracer in the previously constructed 3-D $S$ velocity model based on the travel time tomography (same as shown in Fig. 2). Note that the velocity model may affect the locations of sources. However, we performed the attenuation reconstructions using different velocity models and found that changes in the velocity model have minor effects on the computed attenuation models. An example of the attenuation model derived based on the 1-D velocity distribution is shown in Fig. 5. It can be seen that the locations of sources are slightly different from those in the main model (Fig. 6), which is based on the best 3-D velocity model, but the configuration of the main attenuation patterns looks similar in these two cases. In this sense, the attenuation results appear to be not strongly dependent on the reference velocity models.

The ray coverage was used to construct a parameterization grid with nodes distributed according to the data density. The horizontal grid spacing was $2 \mathrm{~km}$; in the vertical direction, the distances between nodes depended on the ray coverage and were not smaller than $0.5 \mathrm{~km}$. Between the nodes, the velocity was linearly interpolated. To reduce the grid dependency of the results, we performed the inversion for four grids with different basic orientations, then averaged the computed models, and then computed the average model based on the uniform mesh. The first derivative matrix, $\mathbf{A}_{i j}$, represents the numerically computed effect of unit variation in the $i$ th node upon the cumulative attenuation of the $j$ th ray for rays traced in the 3-D model. This matrix was inverted using the LSQR algorithm (Paige and Saunders, 1982). This algorithm uses only non-zero matrix elements and performs the iterative line-by-line processing of the matrix. This allows solving large linear equation systems using modest computing resources. The solution stability was controlled using an additional matrix block to damp the difference between neighboring nodes, which allows for the smoothing of the model.

The model resolution was checked using the synthetic tests presented in Fig. 7. In the map view, the synthetic model has two positive and two negative anomalies $6 \times 6 \mathrm{~km}$ in size that change signs at a depth of $6 \mathrm{~km}$. The inversion provides a fair reconstruction of the general locations of these anomalies; however, their outside contours look deformed, and there is some leakage of anomalies to the initially homogeneous areas. Such distortions should be accounted for when interpreting the observed data inversion results. Notably, the attenuation tomography provides a much more stable reconstruction than expected when using travel time data from passive sources. 

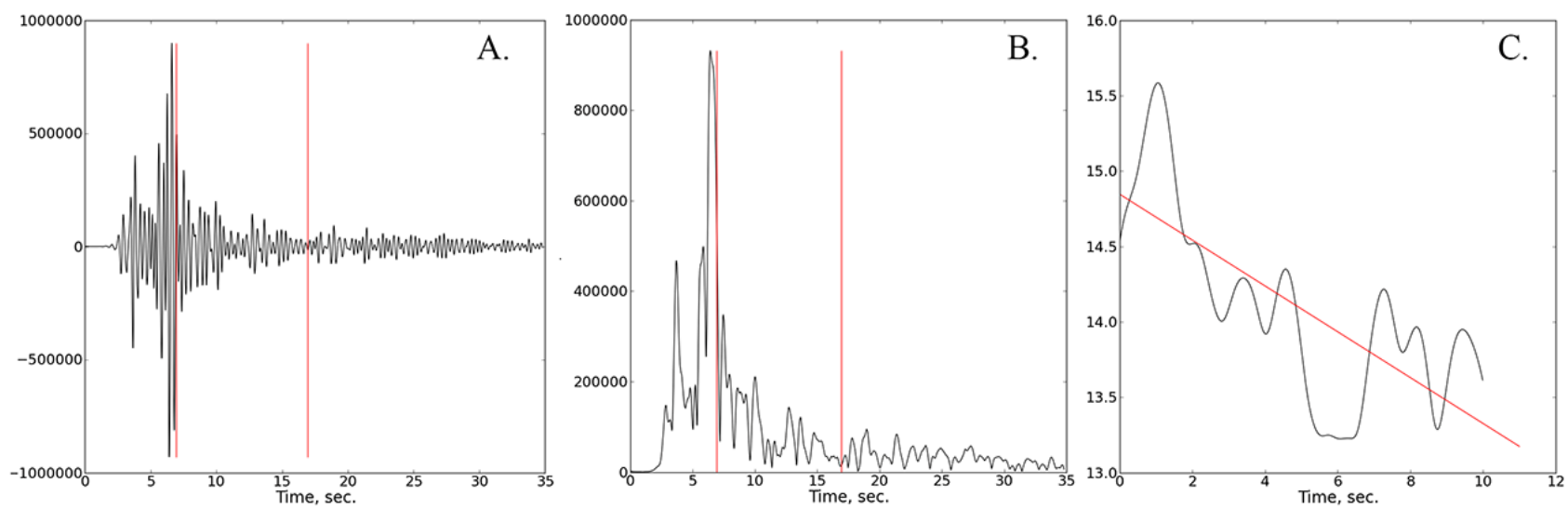

Figure 4. Workflow for the data analysis. Initial seismogram is narrow-band filtered at $3 \mathrm{~Hz}$ (a). The red lines indicate the analyzed time interval. An envelope of absolute deviations in the seismogram (b). Logarithmic transformation of the envelope (c). The red line indicates the linear approximation with the best fit. The slope of this line was then converted into the quality factor.
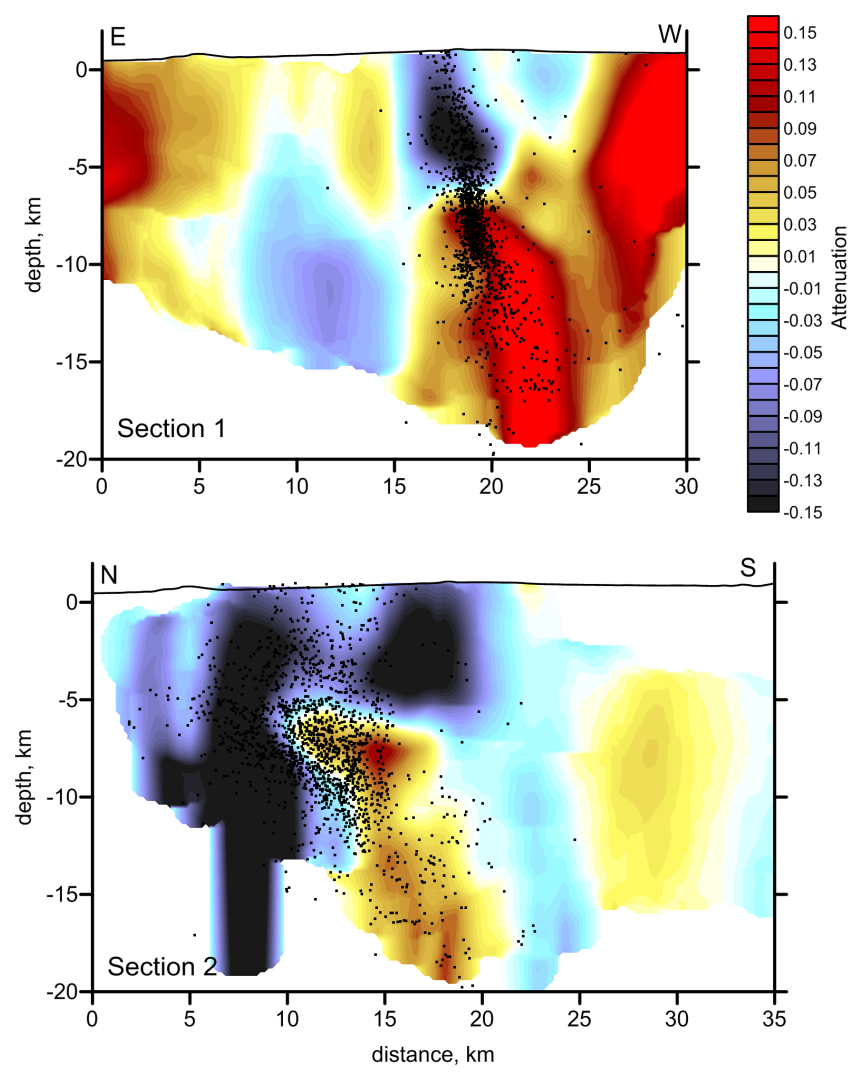

Figure 5. Attenuation distributions and source locations in vertical sections based on the 1-D velocity model. This model can be compared with the main model in Fig. 6 derived in the 3-D model. Locations of the profiles are shown in Fig. 6.

\section{Discussion of the results}

A comparison of the results obtained using three different frequencies is presented in Fig. 8. It can be seen that, for the shallower section, the results at different frequencies appear to be fairly similar: in the central part we observe a low-attenuation anomaly surrounded by prominent highattenuation patterns. For the deeper section, the difference appears to be more important. It might be partly due to different sensitivity of attenuation parameters at different frequencies. It should also be noted that, based on the visual analysis of data, the quality of attenuation retrieval at $3 \mathrm{~Hz}$ was much higher than in the cases of other frequencies. That is why the model corresponding to $3 \mathrm{~Hz}$ frequency was selected as the main result of this study.

Figure 6 shows the resulting attenuation distributions in the vertical and horizontal sections based on data filtered at $3 \mathrm{~Hz}$. For reference, the basaltic flow limits are shown in the horizontal sections. The most reliable results were obtained in the northern part of the study area; the anomalies in the southernmost parts should be interpreted carefully. This result can be compared with seismic velocities obtained earlier. It is hard to make the comparison with the $P$ velocity model computed by Hansen et al. (2013) because they only presented absolute velocities in parallel vertical S-N sections located close to each other. In Sect. 2, which roughly correspond to these profiles, our model demonstrates a transition from low attenuation to the north to high attenuation to the south. This seems to be consistent with high- and lowvelocity anomalies in the Hansen et al. (2013) model. Much clearer correspondence is observed with the velocity model by Koulakov et al. (2014), which is presented in Fig. 2. Especially good fit with the attenuation is achieved for the $\mathrm{Vp} / \mathrm{Vs}$ ratio which is most sensitive to the distributions of fluids and melts. The areas of higher $\mathrm{Vp} / \mathrm{Vs}$ ratio generally match to the areas of high attenuation and vice versa. The consistency of these models confirms the robustness of the derived seismic models.

The attenuation patterns appear to match with the some geological units. For the $2 \mathrm{~km}$ deep shallow section, the 

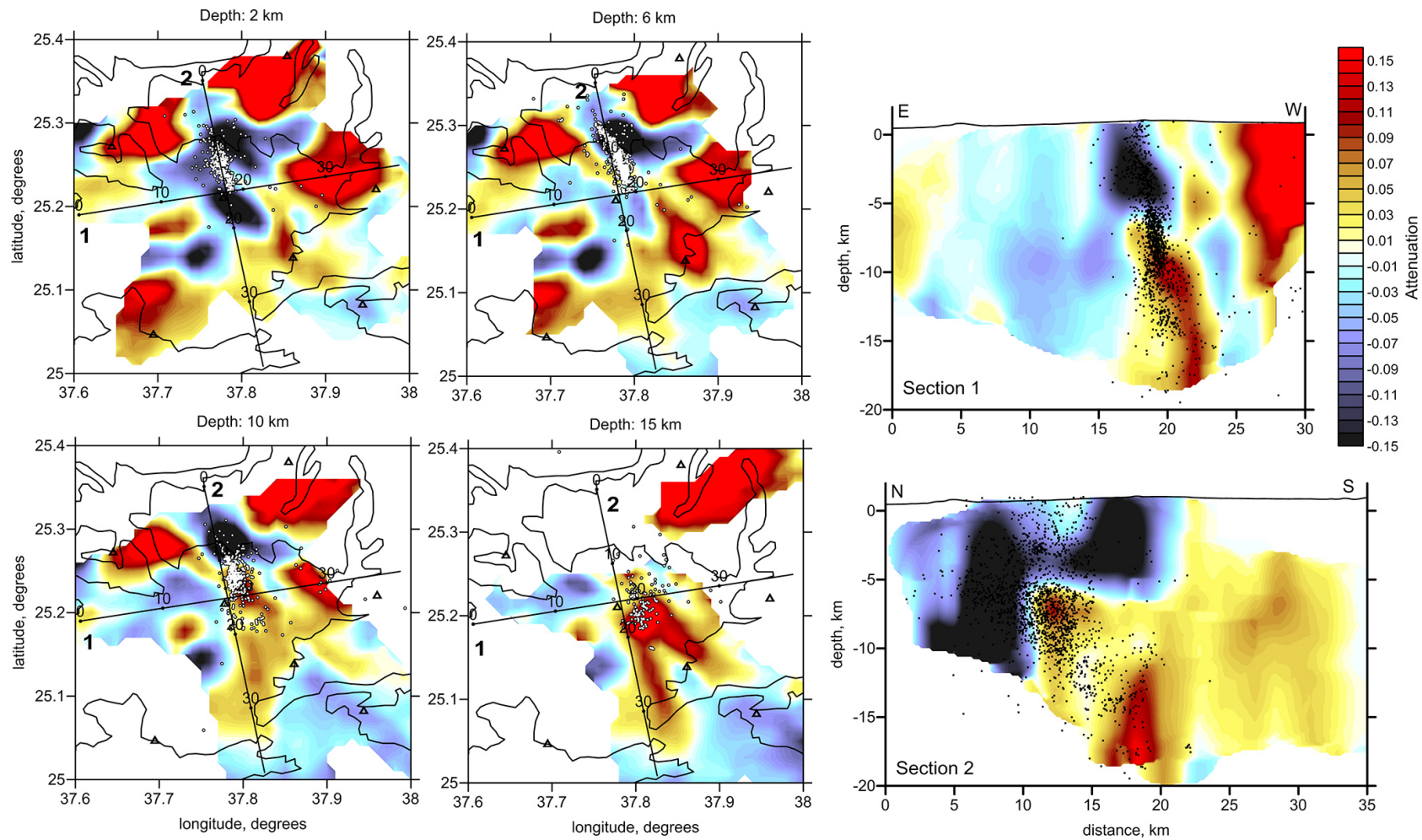

Figure 6. Resulting 3-D distribution of the attenuation in four horizontal and two vertical sections. The dots indicate seismic events near the sections. The triangles are the seismic stations. The black line is the boundary of the basaltic field.

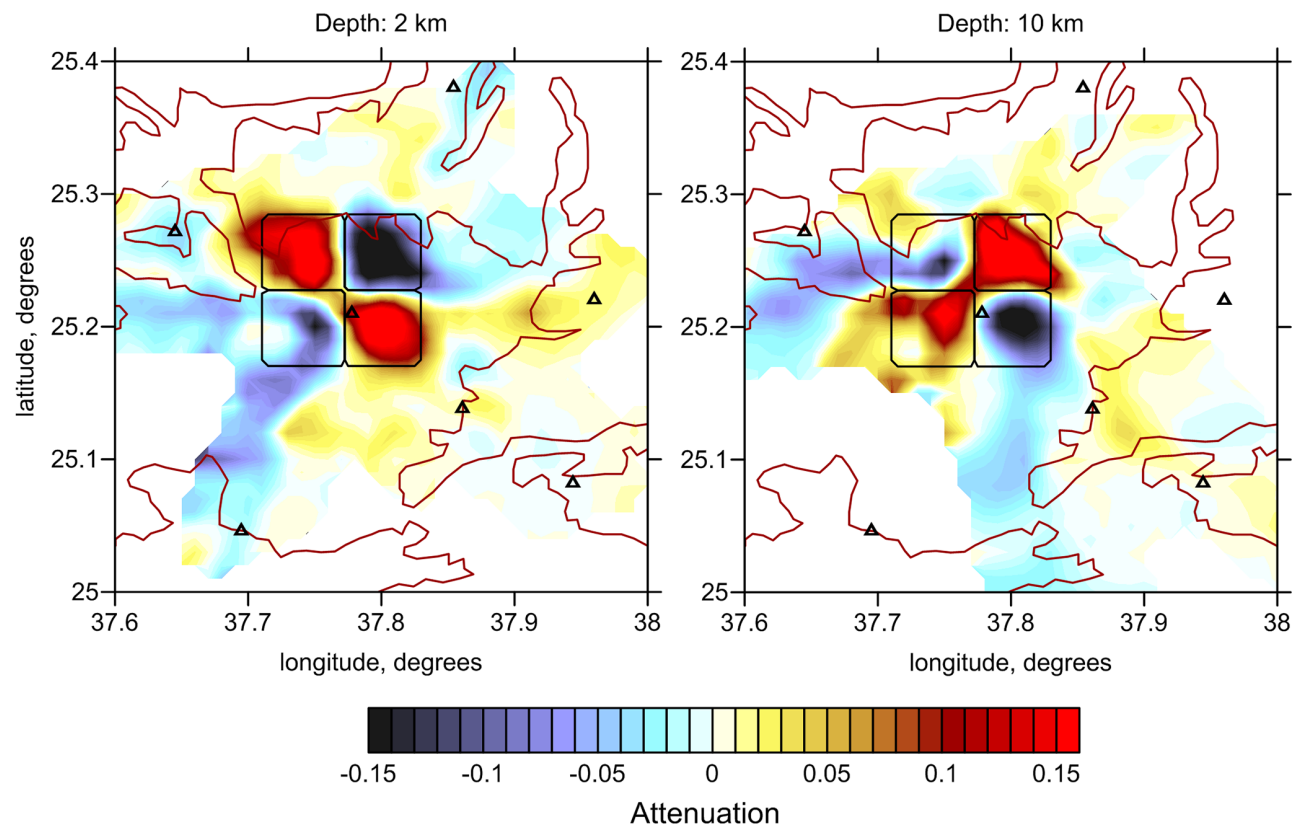

Figure 7. Results of synthetic modeling for the attenuation tomography. Black lines indicate the limits of the synthetic anomalies. At $6 \mathrm{~km}$ depth, the anomalies change the sign. Triangles indicate the seismic stations. The contour of the basalt fields is given by maroon lines for the reference. 

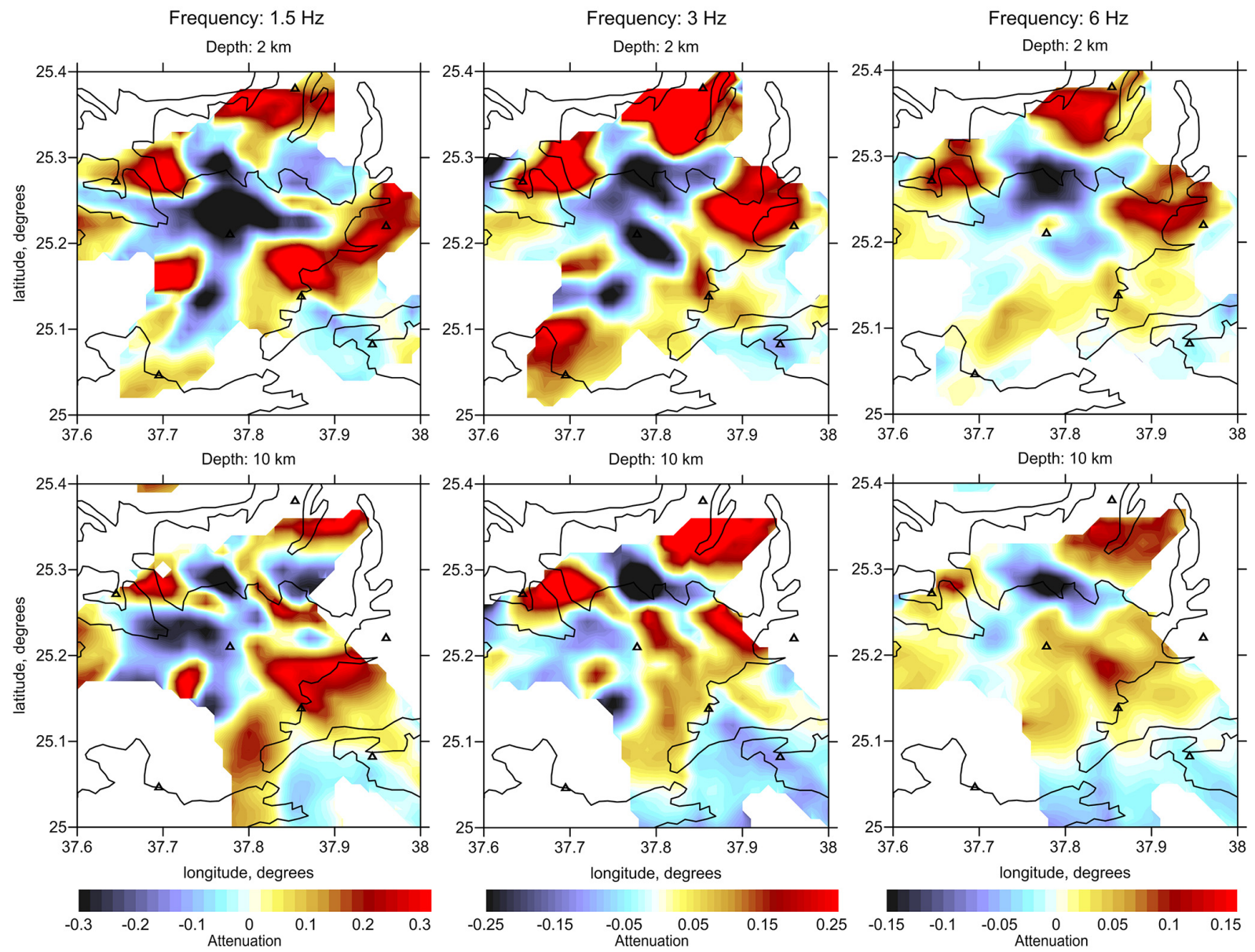

Figure 8. Comparison of the inversion results obtained for the data corresponding to three frequencies: $1.5,3$, and $6 \mathrm{~Hz}$. The results are presented for $2 \mathrm{~km}$ and $10 \mathrm{~km}$ depth. The triangles are the seismic stations. The black line is the boundary of the basaltic field.

northern boundary of the basaltic field corresponds to the transition between high and low attenuations. Areas with low attenuation (high-quality factors) correspond to rigid igneous rocks in the harrat, whereas "red" high-attenuation areas contain softer sediments. Although the high-attenuation areas to the east and south of the study area are less robustly resolved, they also tend to be located near the boundary of or outside the basaltic field.

In the $10 \mathrm{~km}$ deep section, the basaltic field boundary transitions remain unchanged from the shallow section. However, the brightest feature at this depth was a prominent, highattenuation anomaly that coincides with the seismicity distribution. Note that the elongated "tail" of this anomaly expanded to the SE direction possibly caused by radial smearing which may disturb the model, as seen in the synthetic tests (Fig. 7). We see that the upper limit of this highattenuation anomaly is approximately at $6 \mathrm{~km}$ depth for both vertical sections. The synthetic tests using the checkerboard models have a sign transition at the same depth, which indicates that these depth estimates are reliable. In the W-E cross section, the seismicity in the narrow cluster is mostly located within this anomaly; however, a portion of it expands into the upper low-attenuation area. In the N-S cross section, the earthquake distribution is more scattered; however, the lower limit of the seismicity cluster clearly dips southward. In the northern part, most of events occur above $7 \mathrm{~km}$ in depth, while the events observed in the southern part occur at depths of $20 \mathrm{~km}$ and below. This section shows the general fit for the lower limit of the seismicity clusters with high attenuation.

We propose that the local high-attenuation anomaly beneath the central part of the study area may represent a conduit zone that can bring fluids and melts from deeper sources. The seismicity during the Lunayyir crisis in 2009 may have been triggered by ascending fluids and/or melts reaching the bottom of the rigid, uppermost layer of the solid basaltic rocks. The presence of some seismicity in this lowattenuation layer above the transition boundary indicates that the pressure in the conduit zone produced cracks in the upper layer but was insufficient to fracture the layer to the surface. It can be seen that the seismicity stopped at an approximate depth of $2 \mathrm{~km}$. It may be speculated that the rigid cover 
prevented a new eruption in the Harrat Lunayyir. However, the facts all indicate the magma sources are still active and that these sources may find a weaker part of the crust in the future to create a conduit to the surface.

\section{Conclusions}

We constructed a 3-D model of the seismic attenuation distribution in the crust beneath the Harrat Lunayyir. Although less attenuation data were used compared to the travel time data used by Hansen et al. (2013), we achieved a sufficient resolution thanks to the absence of typical trade-offs between the source and model parameters existing for passive source travel time tomography.

The derived model clearly links to the observed geological features and allows the interpretation for the recent seismicity crisis in the Harrat Lunayyir. In the shallow layers, we identified the contrast between the low-attenuation layer within the basalt field and the high-attenuation zones in the surrounding the sedimentary basins. Below $6 \mathrm{~km}$, we found a prominent high-attenuation zone that perfectly confines the narrow seismicity cluster observed in 2009 . We propose that this high-attenuation pattern represents the upper portion of a conduit bringing fluids and/or melts from deep sources. Based on the derived attenuation model and seismicity distribution analyses, we conclude that this conduit most likely reached the depth of $6 \mathrm{~km}$ corresponding to the bottom of a rigid layer which is composed of basalts erupted during previous eruptions. The elevated pressure caused by the conduit process might create cracks within the rigid layer; however, it was insufficient to fracture fully to the surface. In this sense, this basaltic lid saved the area from a new eruption that may occur if the conduit discovers a weaker part of the crust to ascend.

The accumulated seismic records contain significant information on the seismic properties of this activated area, and they are still not fully analyzed. Deriving the $S$ velocity model and assessing the $\mathrm{Vp} / \mathrm{Vs}$ distribution will be particularly important and is the next step of our study.

Acknowledgements. The authors extend their appreciation to the Deanship of Scientific Research at King Saud University (Saudi Arabia) for funding the work through the international research group project no. IRG14-21. Special thanks to the Saudi Geological Survey (SGS) for providing waveform data for this study. The work of the Russian participants is supported by grant RSF no. 14-17-00430. We are grateful to the anonymous reviewer for constructive criticism and wise recommendations.

Edited by: K. Liu

\section{References}

Aki, K.: Scattering and attenuation of shear waves in the lithosphere, J. Geophys. Res., 85, 6496-6504, 1980.

Aki, K. and Chouet, B.: Origin of coda waves: source, attenuation, and scattering effects, J. Geophys. Res., 80, 3322-3342, 1975.

Al-Amri, A. M. S.: Recent seismic activity in the northern Red Sea, J. Geodyn., 20, 243-253, 1995.

Al-Damegh, K., Sandvol, E., Al-Lazki, A., and Barazangi, M.: Regional seismic wave propagation ( $\mathrm{Lg}$ and $\mathrm{Sn}$ ) and $\mathrm{Pn}$ attenuation in the Arabian plate and surrounding regions, Geophys. J. Int., 157, 775-795, 2004.

Al-Dayel, M.,: Geothermal resources in Saudi Arabia, Geothermics, 17, 465-476, 1988.

Baer, G. and Hamiel Y.: Form and growth of an embryonic continental rift: InSAR observations and modeling of the 2009 western Arabia rifting episode, Geophys. J. Int., 182, 155-167, 2010.

Camp, V. E., Hooper, P. R., Roobol, M. J., and White, D. L.: The Madinah eruption, Saudi Arabia: Magma mixing and simultaneous extrusion of three basaltic chemical types, Bull. Volcanol., 49, 489-508, 1987.

Chang, S.J., Merino, M., Van der Lee, S., Stein, S., and Stein, C. A.: Mantle flow beneath Arabia offset from the opening Red Sea, Geophys. Res. Lett., 38, L04301, doi:10.1029/2010GL045852, 2011.

Cochran, J. and Martinez, F.: Structure and tectonics of the northern Red Sea: catching a continental margin between rifting and drifting, Tectonophys., 150, 1-32, 1988.

Cochran, J. R. and Karner, G. D.: Constraints on the deformation and rupturing of continental lithosphere of the Red Sea, in Imaging, Mapping, and Modeling Continental Lithosphere Extension and Breakup, edited by: Karner, G. D., Manatschal, G., and Pinheiro, L. M., Geol. Soc. Publ., 282, 265-289, 2007.

El-Isa, Z. H., and Al-Shanti, A.: Seismicity and tectonics of the Red Sea and western Arabia, Geophys. J., 97, 449-457, 1989.

Evans, J. R. and Zucca, J. J.: Active highresolution seismic tomography of compressional wave velocity and attenuation structure at Medicine Lake Volcano, Northern California Cascade Range, J. Geoph. Res. (1978-2012), 93, 15016-15036, 1988.

Gusev, A. A. and Abubakirov, I. R.: Vertical profile of effective turbidity reconstructed from broadening of incoherent body wave pulses - I. General approach and the inversion procedure, Geophys. J. Int., 136, 295-308, 1999.

Hansen, S., Thurber, C., Mandernach, M., Haslinger, F., and Doran, C.: Seismic velocity and attenuation structure of the east rift zone and south flank of Kilauea Volcano, Hawaii, Bull. Seismol. Soc. Am., 94, 1430-1440, 2004.

Hansen, S. E., DeShon, H. R., Moore-Driskell, M. M., and Al-Amri, A.: Investigating the $\mathrm{P}$ wave velocity structure beneath Harrat Lunayyir, northwestern Saudi Arabia, using double-difference tomography and earthquakes from the 2009 seismic swarm, J. Geophys. Res., 118, 4814-4826, 2013.

Koulakov I.: LOTOS code for local earthquake tomographic inversion. Benchmarks for testing tomographic algorithms, BSSA, 99, 194-214, 2009.

Koulakov, I.: Studying deep sources of volcanism using multiscale seismic tomography, J. Volcanol. Geotherm. Res., 257, 205-226, 2013.

Koulakov, I., Sobolev S. V., and Asch G.: $P$ and $S$ velocity images of the lithosphere-asthenosphere system in the Central An- 
des from local-source tomographic inversion, Geophys. Journ. Int., 167, 106-126, 2006.

Koulakov, I., Bindi, D., Parolai, S., Grosser, H., and Milkereit, C.: Distribution of Seismic Velocities and Attenuation in the Crust Beneath the North Anatolian Fault (Turkey) from Local Earthquake Tomography, Bull. Seism. Soc. Am., 100, 207-224, 2010.

Koulakov, I., El Khrepy, S., Al-Arifi, N., Kuznetsov, P., and Kasatkina, E.: Images of the active magma sources beneath the Lunayyir basaltic field (Saudi Arabia) from local earthquake seismic tomography, Earth Plan. Sci. Lett., 2014.

Lees, J. M.: Seismic tomography of magmatic systems, J. Volcanol. Geotherm. Res., 167, 37-56, 2007.

Lees, J. M. and Lindley, G. T.: Three-dimensional attenuation tomography at Loma Prieta: Inversion of $t^{*}$, Q. J. Geoph. Res., 99, 6843-6863, 1994.

Martínez-Arévalo, C., Patanè, D., Rietbrock, A., and Ibánez, J. M.: The intrusive process leading to the Mt. Etna 2001 flank eruption: Constraints from 3-D attenuation tomography, Geophys. Res. Lett., 32, L21309, doi:10.1029/2005GL023736, 2005.

Paige, C. C. and Saunders, M. A.: LSQR: An algorithm for sparse linear equations and sparse least squares, ACM Trans. Math. Soft, 8, 43-71, 1982.
Pallister, J. S., McCausl, W. A., Jónsson, S., Lu Z. H. M., El Hadidy, S., Aburukbah, A., Stewart, I. C. F., Lundgren, P. R., White, R. A., and Moufti, M. R. H.: Broad accommodation of riftrelated extension recorded by dyke intrusion in Saudi Arabia, Nat. Geosci. 3. 705-712, 2010.

Rautian, T. G. and Khalturin, V. I.: The use of the coda for determination of the earthquake source spectrum, Bull. Seismol. Soc. Am., 68, 923-948, 1978.

Sato, H. and Fehler M. C.: Seismic Wave Propagation and Scattering in the Heterogeneous Earth, AIP, New York, 308 pp., 1998.

Schurr, B., Asch, G., Rietbrock, A., Trumbull, R., and Haberland, C. H.: Complex patterns of fluid and melt transport in the central Andean subduction zone revealed by attenuation tomography, Earth Planet. Sci. Lett., 215, 105-119, 2003.

Zobin, V. M., Al-Amri, A. M., and Fnais, M.: Seismicity associated with active, new-born, and re-awakening basaltic volcanoes: case review and the possible scenarios for the Harraat volcanic provinces, Saudi Arabia, Arab. J. Geosci., 6, 529-541, 2013. 